EPJ Web of Conferences 13, 06003 (2011)

DOI: $10.1051 /$ epjconf/20111306003

(c) Owned by the authors, published by EDP Sciences, 2011

\title{
HBT radii from the UrQMD transport approach at different energies
}

\author{
Gunnar Gräf ${ }^{1,2, a}$, Elliot Mount ${ }^{3}$, Hannah Petersen ${ }^{4}$, Qingfeng $\mathrm{Li}^{5}$, Mike Lisa ${ }^{3}$, and Marcus Bleicher ${ }^{1,2}$ \\ 1 Frankfurt Institute for Advanced Studies (FIAS), Ruth-Moufang-Str. 1, 60438 Frankfurt am Main, Germany \\ 2 Institut für Theoretische Physik, Goethe-Universität, Max-von-Laue-Str. 1, 60438 Frankfurt am Main, Germany \\ 3 Department of Physics, Ohio State University, Columbus, Ohio 43210, United States \\ 4 Department of Physics, Duke University, Durham, North Carolina 27708-0305, United States \\ 5 School of Science, Huzhou Teachers College, Huzhou 313000, P.R. China
}

\begin{abstract}
We present results on Hanbury Brown-Twiss (HBT) radii extracted from the Ultra-relativistic Molecular Dynamics (UrQMD) approach to relativistic heavy ion collisions. The present investigation provides a comparison of results from pure hadronic transport calculations to a Boltzmann + Hydrodynamic hybrid approach with an intermediate hydrodynamic phase. For the hydrodynamic phase different Equations of State (EoS) have been employed, i.e. bag model, hadron resonance gas and a chiral EoS. The influence of various freeze-out scenarios has been investigated and shown to be negligible if hadronic rescatterings after the hydrodynamic evolution are included. Furthermore, first results of the source tilt from azimuthal sensitive HBT and the direct extraction from the transport model are presented and exhibit a very good agreement with E895 data at AGS.
\end{abstract}

\section{Introduction}

To explore the properties of nuclear matter under extreme conditions one collides heavy ions at relativistic energies. The needed collision energies are achieved by using heavy ion colliders such as the Super Proton Synchrotron (SPS), the Relativistic Heavy Ion Collider (RHIC) and the Large Hadron Collider (LHC) or the soon to be built Facility for Antiproton and Ion Research (FAIR). During the collision of these nuclei it is expected that a hot and dense zone of free quarks and gluons, known as the quark gluon plasma (QGP) is created.

Due to the ultra-short duration of a relativistic heavy ion collision on the order of $10 \mathrm{fm} / \mathrm{c}$ it is impossible to observe the creation and behaviour of the QGP directly. It is only possible to observe the momentum-space distributions of the particles produced in the reaction long after they have hadronized and the unstable hadrons have decayed. Because of this theoretical modelling is needed to reconstruct the phasespace evolution of the medium and to investigate if a phase transition to the QGP state has happened.

Under the name of HBT interferometry comes a powerful theoretical tool, that allows to connect the final particle momentum distribution to the freeze-out geometry. It is however not possible to deduce the full space-time dynamic with this approach. Thus, one uses transport approaches to generate freeze-out distributions that allow to calculate theoretical HBT parameters while at the same time knowing the whole space-time evolution of the particle emitting system.

\footnotetext{
a e-mail: graef@th.physik.uni-frankfurt.de
}

While transport approaches with only hadronic or partonic degrees of freedom allow for a very detailed exploration of the collision dynamics they usually remain either in the hadronic or partonic phase and do not allow for a phase transition. Therefore, it is inherently impossible to directly see signals of a phase transition in such models. The only option within these models is to compare computation results from hadronic calculations with partonic results and watch out for qualitative changes in the behaviour.

In contrast to that it is easily possible to change the degrees of freedom within hydrodynamic descriptions. Here, one has to change the equation of state to model either partonic or hadronic degrees of freedom. It is even possible to use an EoS that features a phase transition, e.g. a bag model EoS. However, before the kinetic freeze-out the medium becomes so dilute, that a hydrodynamic description becomes questionable. To tackle this challenge, hybrid approaches that combine particle transport with hydrodynamic calculations have been developed during the past few years.

In this paper the effects of different EoS and different transition scenarios from the hydrodynamic description to the kinetic transport description on HBT results are explored.

\section{Hybrid approaches}

Hybrid approaches that combine hydrodynamics and transport equations were proposed 10 years ago $[1,2]$. Since then they have been employed for a wide range of investigations [3-6]. The hybrid approach that is used here is 
based on the well known UrQMD model [7-9] with an additional intermediate hydrodynamic evolution. This approach starts with a purely hadronic cascade. After the two nuclei have passed through each other the hydrodynamic evolution starts at the time $t_{\text {start }}=2 R / \sqrt{\gamma^{2}-1}$, with $\mathrm{R}$ being the radius of the nucleus and $\gamma$ being the Lorentz factor. At this time the spectators and the other particles are treated differently. The spectators are still propagated via the cascade, while the other particles are mapped onto the hydrodynamic grid. By doing so one explicitly assumes a local thermodynamic equilibrium for each cell. For the following hydrodynamic evolution the SHASTA [10] algorithm is used. For the analysis in this paper a hadron gas EoS (HG), a bag model EoS (BM) and an EoS with a chiral phase transition $(\mathrm{CH})$ are used. The $\mathrm{HG}$ EoS is modeled with the same degrees of freedom as the UrQMD model $[11,12]$. At the end of the hydrodynamic expansion the hydrodynamic quantities are translated to hadronic degrees of freedom via the Cooper-Frye formalism. For this, two different freeze-out scenarios were employed. The first one is the isochronous freeze-out (IF). In the IF the hydrodynamic evolution stops in all cells at the same time, once the energy density in every cell drops below five times the ground state density $\epsilon_{0}$ (default value). The second one is the gradual freeze-out (GF) scenario. In this scenario the mapping from the hydrodynamic grid to the cascade happens in transverse slices of thickness $0.2 \mathrm{fm}$. All cells of a transverse slice freeze-out, once every cell in this slice has reached an energy density lower than five times the ground state density. This mimics an iso-eigentime freeze-out, that accounts for time dilation effects seen in the freeze-out temperature in cells with a high rapidity. After this the final state interactions and decays happen within the UrQMD framework. For some of the calculations depicted here, these final state interactions have been omitted to gauge the effect of the final rescattering on the examined quantities. For a more detailed portrait of the used model the reader is referred to $[13,14]$.

\section{$3 \mathrm{HBT}$ radii}

To explore the effect of different freeze-out scenarios on the HBT radii we have compared the results for a hydrodynamic evolution using the gradual freeze-out at $5 \epsilon_{0}$ (default) and $4 \epsilon_{0}$ with an isochronous freeze-out and a gradual freeze-out without hadronic rescattering (HR) as explained in Section 2. Without HR means, that the evolution stops immediately after the freeze-out of the hydrodynamic phase and all created resonances decay instantaneously. The transverse momentum $k_{T}\left(\mathbf{k}_{t}=\left(\mathbf{p}_{1 T}+\mathbf{p}_{2 T}\right) / 2\right.$ ) dependence of the HBT radii for these calculations are plotted in Fig. 1. All the radii were calculated using the correlation afterburner (CRAB) $[15,16]$ by Scott Pratt. As one can see, the results without HR are significantly different from the ones with rescattering. If one includes HR the radii increase and the data is slightly, but consistently overpredicted by all freeze-out scenarios. The HR has a major effect on the HBT results. Once the HR has been included,

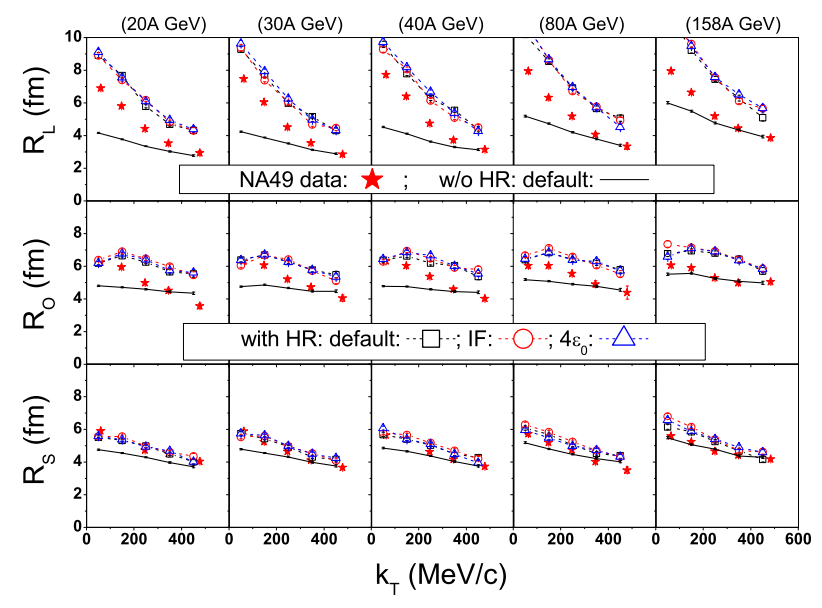

Fig. 1. $k_{T}$ dependence of $\pi^{-}$HBT radii at beam energies from $20 \mathrm{AGeV}$ to $160 \mathrm{AGeV}$ for different freeze-out scenarios [14]. The red stars are NA49 data [17]. All other results in this plot are from hybrid calculations. The black squares depict the gradual freeze-out (GF) at 5 times $\epsilon_{0}$ (default scenario). The black line is the same scenario, but without hadron rescattering (HR) after the hydrodynamic evolution. The red circles are for the isochronous freeze-out (IF). And the blue triangles for GF at 5 times $\epsilon_{0}$.

all the differences in the freeze-out treatments vanish and the different methods become indistinguishable.

An analysis of the different equations of state is shown in Fig. 2. Here the same experimental data as in Fig. 1 is shown. The theoretical results are for $\mathrm{Pb}+\mathrm{Pb}$ collisions with the pure hadronic cascade and with the $\mathrm{HG}, \mathrm{BM}$ and $\mathrm{CH}$ EoS. The cascade provides fairly good results for $R_{S}$ and $R_{L}$ while it slightly over predicts the data for $R_{O}$. The results from the hybrid model calculations have for all EoS in common, that they overpredict the data especially for $R_{O}$ and $R_{L}$. While the $\mathrm{CH}$ and the HG EoS lead to very similar results, the values of the BM increase even more. We attribute this additional increase to the large latent heat present in the bag model that drives the interactions in the late stage of the collision.

In Fig. 3 the $R_{\text {out }}$ over $R_{\text {side }}$ ratio is shown for all EoS (HG,BM,CH) and freeze-out scenarios (IF,GF,no HR) and for UrQMD 2.3 in cascade mode. This ratio is dependent on the lifetime of the observed system and should increase with increasing lifetime. It has been predicted [18], that a strong first order phase transition leads to a significant increase in the $R_{\text {out }}$ over $R_{\text {side }}$ ratio due to the existence of an intermediate mixed phase with hadronic and partonic degrees of freedom that delays the expansion of the system. Contrary to the prediction [18] no prominent peak emerges in the $R_{\text {out }}$ over $R_{\text {side }}$ ratio in Fig. 3 although the BM EoS includes a first order phase transition. However the hybrid results show differences for the different EoS and the BM EoS exhibits an increase in the $R_{\text {out }}$ over $R_{\text {side }}$ ratio with a maximum in the mid-SPS energy regime. On the other hand the results are insensitive to the studied freeze-out scenarios if hadronic rescattering is included. 


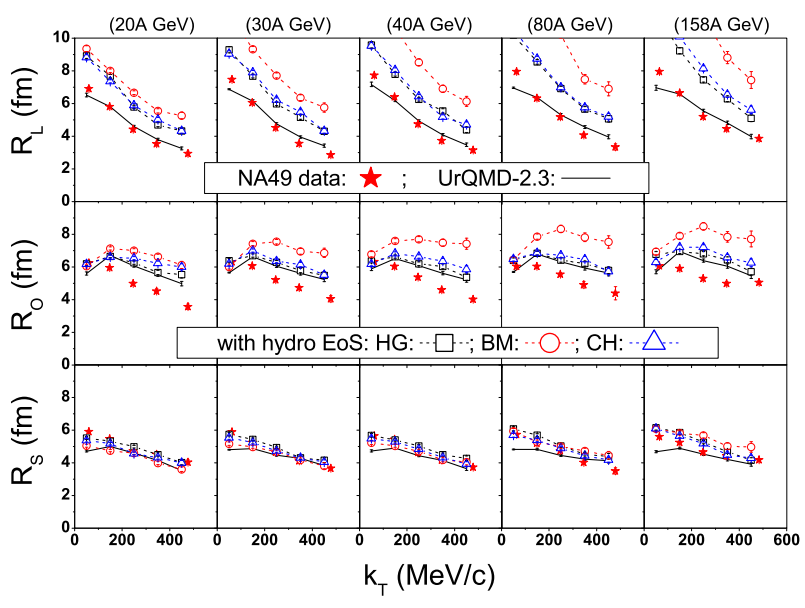

Fig. 2. $k_{T}$ dependence of $\pi^{-}$HBT radii at beam energies from 20 $\mathrm{AGeV}$ to $160 \mathrm{AGeV}$ for different Equations of state [14]. The red stars are NA49 data [17]. Black lines correspond to the standard UrQMD calculation without hydrodynamic phase. All other results in this plot are from hybrid calculations. The black squares depict the Hadron Gas EoS (HG), the red circles stand for the bag model (BM) EoS and the blue triangles show the results for the EoS with chiral phase transition $(\mathrm{CH})$.

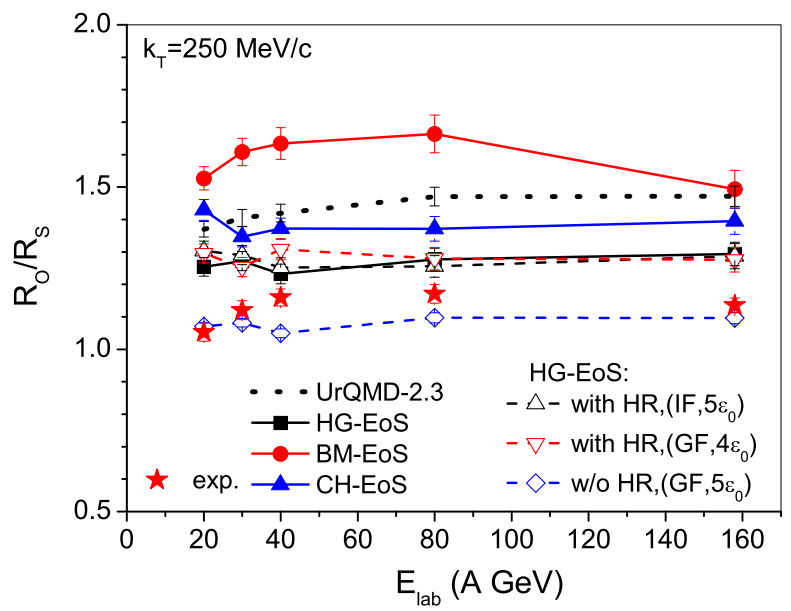

Fig. 3. Excitation function of the $\pi^{-} R_{O} / R_{S}$ ratio for a transverse momentum of $k_{T}=250 \mathrm{MeV} / \mathrm{c}$. The red stars depict NA49 data [17]. The full symbols stand for the hybrid calculations with HG, $\mathrm{BM}$ and $\mathrm{CH}$ EoS, while the empty symbols describe the different freeze-out scenarios for the HG EoS. The dotted line represents the cascade results.

\section{Source Tilt}

For non-central heavy ion collisions only a part of the colliding nuclei is stopped. For geometrical reasons the collision zone is of almond shape if one looks along the beam axis. An often neglected fact is that the zone of stopped matter is tilted with respect to the collision axis, due to the different movement directions of the colliding nuclei (See Fig. 4). It is possible to extract the tilt angle $\theta_{s}$ either di-

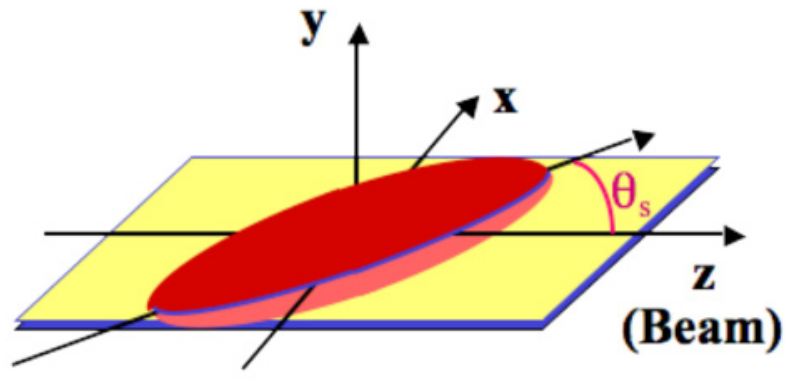

Fig. 4. Schematic picture of a non-central heavy ion collision depicting the source tilt with respect to the beam axis.

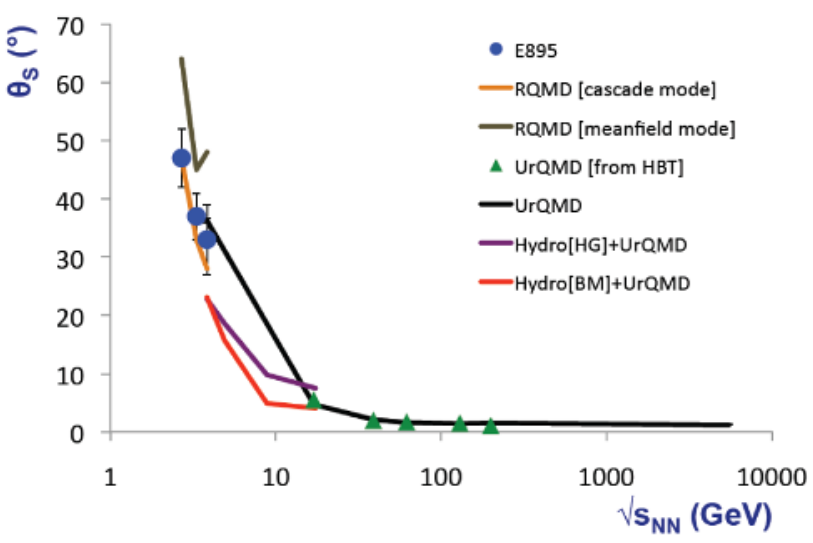

Fig. 5. Energy dependence of the tilt angle $\theta_{S}$ in non central heavy ion collisions [19]. The different lines correspond to results gained directly from the freeze-out coordinates, either from UrQMD in Hydro (HG \& BM EoS) and cascade mode or from RQMD. The triangles depict UrQMD cascade mode results achieved using azimuthal sensitive HBT. The circles are data from E895 [20].

rectly from the freeze-out coordinates if one is in a theoretical framework or from an azimuthal sensitive HBT analysis [20,21]. A theoretical model gives the opportunity to compare the results of both methods, which has been done for Fig. 5. The figure shows the tilt angle in dependence of the center of mass energy. There are several noteworthy points in Fig. 5. The first one is that one observes good agreement between the RQMD and the UrQMD model. At the same time both models describe the E895 data, which gives confidence for the calculation of the tilt angle. The second one is that there is a very good agreement between the tilt angle extracted via fits to the freeze-out coordinates (lines) and the tilt angle calculated via the azimuthal sensitive HBT radii (triangles). The third one is that the tilt decreases with increasing collision energies. This tells us, that it is very important to take the tilt into account if one computes tilt dependent quantities like the elliptic flow or the source eccentricity at AGS and SPS energies. At RHIC and LHC energies however it is no problem to neglect the tilt because it vanishes due to the trivial geometrical effect of the elongation of the collision zone along the beam axis at these high energies. 
In summary we have shortly presented a hybrid hydrodynamic model within the UrQMD framework. Our emphasis was on the study of the effect of different equations of state and freeze-out scenarios on the Hanburry BrownTwiss correlations. We showed that $R_{\text {out }}$ and $R_{\text {long }}$ were generally overpredicted if the intermediate hydrodynamic evolution was activated especially with the bag model EoS. Furthermore it was observed that in all investigated freezeout prescriptions the differences in the final state are washed out if hadron rescattering is taken into account. Within the studies of the $R_{\text {out }}$ over $R_{\text {side }}$ ratio no prominent sign of a phase transition is present even for EoS with phase transitions. The last point was, that the source tilt is significant and not to be neglected in non-central heavy-ion collisions at AGS and low SPS energies.

\section{Acknowledgements}

This work was supported by the Helmholtz International Center for FAIR within the framework of the LOEWE program launched by the State of Hesse, GSI, BMBF and DESY. The hydrodynamic model has been provided by Dirk Rischke. The calculations have been computed at the Center for Scientific Computing at Goethe University. For the calculations of the HBT radii the correlation afterburner (CRAB) of Scott Pratt was used. Q.Li thanks the supports by the key project of MEC (No. 209053), the NSFC (Nos. 10905021,10979023), the Zhejiang-NSFC (No.Y6090210), and the Qian-Jiang Talents Project of Zhejiang Province (No. 2010R10102). H.P. acknowledges a Feodor Lynen fellowship of the Alexander von Humboldt foundation.

\section{References}

1. S. A. Bass, A. Dumitru, M. Bleicher, L. Bravina, E. Zabrodin, H. Stoecker and W. Greiner, Phys. Rev. C 60, 021902 (1999) [arXiv:nucl-th/9902062].

2. A. Dumitru, S. A. Bass, M. Bleicher, H. Stoecker and W. Greiner, Phys. Lett. B 460, 411 (1999) [arXiv:nuclth/9901046].

3. D. Teaney, J. Lauret and E. V. Shuryak, arXiv:nuclth/0110037.

4. O. J. Socolowski, F. Grassi, Y. Hama and T. Kodama, Phys. Rev. Lett. 93, 182301 (2004) [arXiv:hep$\mathrm{ph} / 0405181]$.

5. C. Nonaka and S. A. Bass, Nucl. Phys. A 774, 873 (2006) [arXiv:nucl-th/0510038].

6. T. Hirano and M. Gyulassy, Nucl. Phys. A 769, 71 (2006) [arXiv:nucl-th/0506049].

7. H. Petersen, M. Bleicher, S. A. Bass and H. Stocker, arXiv:0805.0567 [hep-ph].

8. M. Bleicher et al., J. Phys. G 25 (1999) 1859 [arXiv:hep-ph/9909407].

9. S. A. Bass et al., Prog. Part. Nucl. Phys. 41 (1998) 255 [Prog. Part. Nucl. Phys. 41 (1998) 225] [arXiv:nuclth/9803035].
10. D. H. Rischke, S. Bernard and J. A. Maruhn, Nucl. Phys. A 595, 346 (1995) [arXiv:nucl-th/9504018].

11. L. V. Bravina et al., J. Phys. G 25 (1999) 351 [arXiv:nucl-th/9810036].

12. L. V. Bravina et al., Phys. Rev. C 63 (2001) 064902 [arXiv:hep-ph/0010172].

13. H. Petersen, J. Steinheimer, G. Burau, M. Bleicher and H. Stocker, Phys. Rev. C 78, 044901 (2008) [arXiv:0806.1695 [nucl-th]].

14. Q. Li, J. Steinheimer, H. Petersen, M. Bleicher and H. Stocker, Phys. Lett. B 674 (2009) 111 [arXiv:0812.0375 [nucl-th]].

15. S. Pratt, CRAB version 3, http://www.pa.msu.edu/ pratts/freecodes/crab/home.html

16. S. Pratt, J. Sullivan, H. Sorge et al., Nucl. Phys. A566 (1994) 103C-114C.

17. C. Alt et al. [ NA49 Collaboration ], Phys. Rev. C77, 064908 (2008). [arXiv:0709.4507 [nucl-ex]].

18. D. H. Rischke, M. Gyulassy, Nucl. Phys. A608 (1996) 479-512. [nucl-th/9606039].

19. E. Mount, G. Gräf, M. Mitrovski, M. Bleicher and M. Lisa, arXiv:1012.5941 [nucl-th].

20. M. A. Lisa et al. [E895 Collaboration], Phys. Lett. B 496, 1 (2000) [arXiv:nucl-ex/0007022].

21. M. A. Lisa, U. W. Heinz and U. A. Wiedemann, Phys. Lett. B 489 (2000) 287 [arXiv:nucl-th/0003022]. 